\title{
Monitoring and evaluation of disaster response efforts undertaken by local health departments: a rapid realist review
}

\author{
Kate Gossip ${ }^{1,2^{*}}$, Hebe Gouda ${ }^{1,2}$, Yong Yi Lee ${ }^{1,2}$, Sonja Firth ${ }^{3}$, Raoul Bermejo III ${ }^{4,5}$, Willibald Zeck ${ }^{4,6}$
} and Eliana Jimenez Soto ${ }^{7}$

\begin{abstract}
Background: Local health departments are often at the forefront of a disaster response, attending to the immediate trauma inflicted by the disaster and also the long term health consequences. As the frequency and severity of disasters are projected to rise, monitoring and evaluation (M\&E) efforts are critical to help local health departments consolidate past experiences and improve future response efforts. Local health departments often conduct M\&E work post disaster, however, many of these efforts fail to improve response procedures.

Methods: We undertook a rapid realist review (RRR) to examine why M\&E efforts undertaken by local health departments do not always result in improved disaster response efforts. We aimed to complement existing frameworks by focusing on the most basic and pragmatic steps of a M\&E cycle targeted towards continuous system improvements. For these purposes, we developed a theoretical framework that draws on the quality improvement literature to 'frame' the steps in the M\&E cycle. This framework encompassed a M\&E cycle involving three stages (i.e., document and assess, disseminate and implement) that must be sequentially completed to learn from past experiences and improve future disaster response efforts. We used this framework to guide our examination of the literature and to identify any context-mechanism-outcome $(\mathrm{CMO})$ configurations which describe how M\&E may be constrained or enabled at each stage of the M\&E cycle.

Results: This RRR found a number of explanatory CMO configurations that provide valuable insights into some of the considerations that should be made when using M\&E to improve future disaster response efforts. Firstly, to support the accurate documentation and assessment of a disaster response, local health departments should consider how they can: establish a culture of learning within health departments; use embedded training methods; or facilitate external partnerships. Secondly, to enhance the widespread dissemination of lessons learned and facilitate inter-agency learning, evaluation reports should use standardised formats and terminology. Lastly, to increase commitment to improvement processes, local health department leaders should possess positive leadership attributes and encourage shared decision making.

(Continued on next page)
\end{abstract}

\footnotetext{
*Correspondence: k.gossip@qcmhr.uq.edu.au

'School of Public Health, The University of Queensland, Level 2, Public Health

Building (887) Corner of Herston Road and Wyndham Street, Herston QLD

4006, Australia

${ }^{2}$ Policy and Epidemiology Group, Queensland Centre for Mental Health

Research, The Park Centre for Mental Health, Level 3, Dawson House, Wacol

QLD 4076, Australia

Full list of author information is available at the end of the article
} 
(Continued from previous page)

Conclusion: This study is among the first to conduct a synthesis of the CMO configurations which facilitate or hinder M\&E efforts aimed at improving future disaster responses. It makes a significant contribution to the disaster literature and provides an evidence base that can be used to provide pragmatic guidance for improving M\&E efforts of local health departments.

Trial registration: PROSPERO 2015:CRD42015023526.

Keywords: Monitoring and evaluation, Disaster response, Health departments, Lessons learned, Rapid realist review

\section{Background}

The Sendai Framework for disaster risk reduction 20152030 notes that natural disasters have caused more than \$US 1.3 trillion worth of damage, affected 1.5 billion people and led to approximately 700,000 deaths over the past decade [1]. Poor, vulnerable and marginalised populations in low and middle income countries (LMICs) of East Asia and the Pacific are among those disproportionately affected by natural disasters [2]. Local health departments are often at the forefront of an emergency response, attending to both the immediate trauma and long term health consequences resulting from a disaster. Evaluating a department's response to a disaster can offer insights into what supports or hinders the successful delivery of health services during a disaster [3].

From an evaluation perspective, substantive work has been undertaken recently to unpack the theory of change behind disaster efforts in general. The most prominent examples are the disaster logic model and the five frameworks developed by Birnbaum and colleagues [4-8]. They provide a systematic approach to assess the how and why disaster interventions lead to specific outcomes. However, very little is known about whether the $M \& E$ of disaster response efforts leads to better outcomes in terms of improved practice.

Monitoring and Evaluation (M\&E) can be defined as a quality improvement process which health departments use to monitor, measure and assess performance. Evaluation reports are intended to identify strengths and weaknesses of a particular response and enable practical lessons to be learned and applied [9, 10]. These lessons feed into future strategic planning and are critical to both: formulating and revising policy; and refining improvement strategies $[10,11]$. That being said, existing evidence demonstrates that lessons are not always learned from past disasters $[4,12-15]$. If this continues to be the case, the priorities for action established by the Sendai Framework will fail to materialise. By not learning from past mistakes, key stakeholders will be unable to: improve their understanding of local disaster risks; strengthen local governance; improve the return on their increased investments; and enhance future disaster response efforts.
Savoia and colleagues [14] found that the same failure to learn lessons from previous disasters occurred during the public health emergency responses to three separate hurricanes in North America (i.e., Katrina 2005, Gustav 2008 and Ike 2008). Despite documentation of the problems, public health response procedures were not revised resulting in the same challenges being experienced years later during the emergency response to the $\mathrm{H} 1 \mathrm{~N} 1$ pandemic in 2009-2010.

This demonstrates that the failure to act on problems identified from past experiences can leave health systems and communities susceptible to the recurrence of the same problems during future events [4, 12-15]. A more recent example of this can be found in the response to Super Typhoon Haiyan, one of the most destructive typhoons recorded, which made landfall in the Philippines on November 2013. Following Haiyan, a stocktaking exercise was undertaken by the Philippine Department of Health and UNICEF to review the health response in the most affected regions. This exercise revealed that a number of provinces shared common challenges in responding to Haiyan, several of which had been experienced in previous disasters. Although the Philippine Department of Health conducted post-incident evaluations after each previous disaster, it was acknowledged that these efforts did not necessarily lead to improved disaster response procedures in the present circumstances (personal communication).

The aim of this study was to gain an understanding of why M\&E efforts undertaken by local health departments often fail to improve future disaster responses. We used a rapid realist review (RRR) approach which adopts a realist perspective to knowledge synthesis to identify context-mechanism-outcome (CMO) configurations. The CMO configurations identified in the literature provide insights into why and how M\&E is likely to work [16]. These findings can be used to help guide local health departments to improve M\&E processes.

\section{Methods \\ Overview}

Our methods followed the six stages of realist evaluation outlined by Pawson [17] [1]: identify the research 
question [2]; formulate a theoretical framework [3]; search for primary studies [4]; select and appraise the quality of studies [5]; extract, analyse and synthesise the relevant data; and [6] refine the theoretical framework. Our research was also guided by the Realist and Metanarrative Evidence Synthesis: Evolving Standards (RAMESES) publication standards [18].

\section{Identification of the research question}

This study was conducted in response to the findings of the stocktaking exercise conducted after super typhoon Haiyan, which highlighted that previous post-disaster evaluations had not led to improvements in existing disaster response procedures. We consulted with a local reference group in the Philippines to clarify the focus of the review and determine what knowledge would be most useful to their efforts to improve future disaster responses. Our primary research question asked: how, why and under what circumstances can local health departments use $M \& E$ interventions to improve future disaster responses based on past experiences? We aimed to explore these questions by [1]: examining the contextual factors operating within M\&E; and [2] identifying and analysing the mechanisms which facilitate or hinder successful M\&E processes.

\section{Formulation of the theoretical framework}

The theoretical framework for this study was developed based on a broad search of peer reviewed and grey literature describing health departments' experiences with M\&E. We formulated a framework based on the three fundamental stages of an M\&E cycle that should be sequentially completed in order to successfully learn from past experiences - i.e., document and assess, disseminate and implement (Fig. 1). We used this framework to guide our examination of the literature and to analyse any constraining or enabling features which influence outcomes at each stage of the M\&E cycle.

Realist evaluation is a theory driven approach which uses context-mechanism-outcome (CMO) configurations to understand the causal patterns underlying the success or failure of an intervention. CMO configurations explain how particular contexts (c) generate casual mechanisms $(\mathrm{m})$ that produce an outcome (o) within an intervention [19]. A brief description of how disaster responses can be understood with respect to each stage within the M\&E cycle is outlined below.

Stage 1 - Document and assess. The first stage of the M\&E cycle is to document and assess the response to the disaster. This involves using a systematic approach to record information during and immediately after the response effort. This information is then reflected upon by personnel who are trained to conduct an assessment of the response. The goal of the assessment is to identify

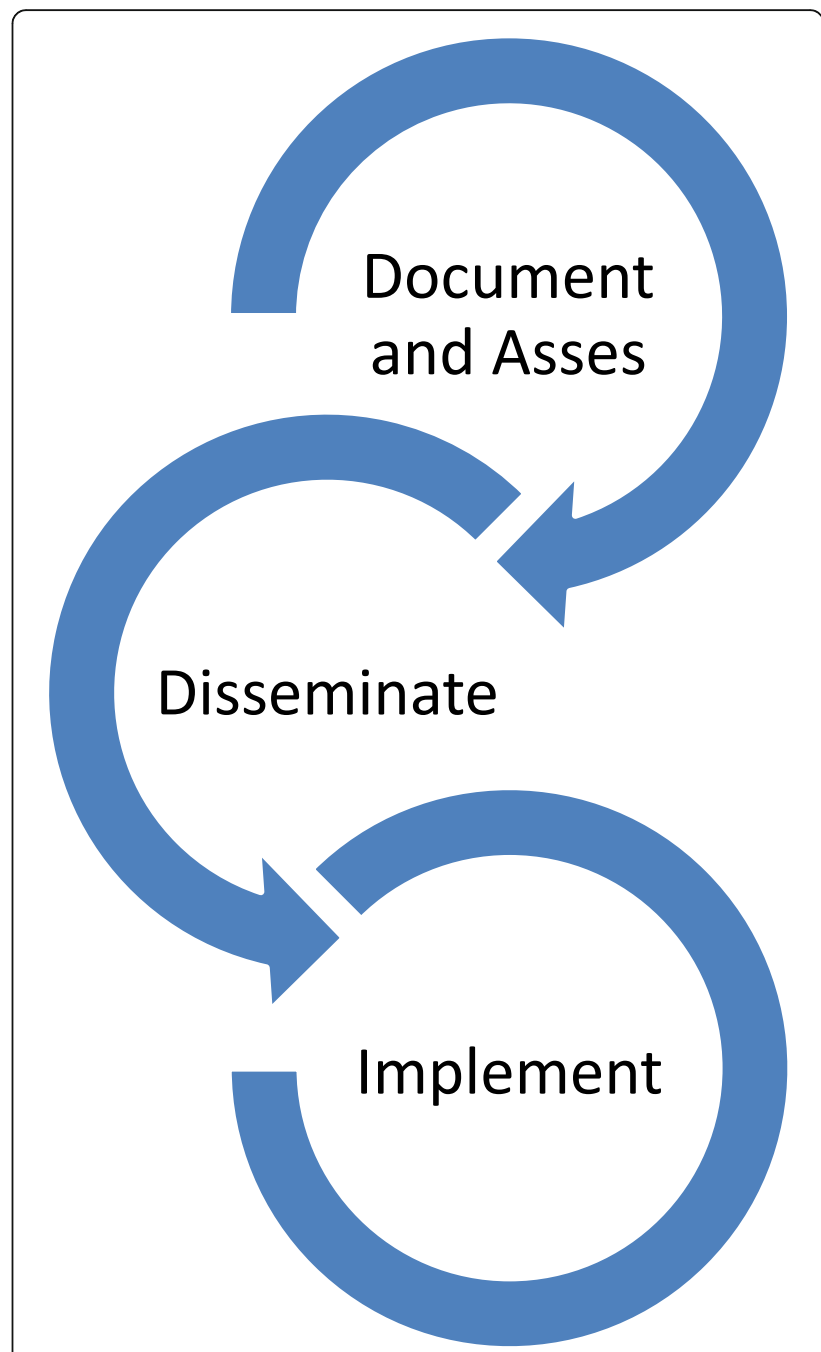

Fig. 1 Monitoring and Evaluation framework

the fundamental strengths and weaknesses of a recent response to a natural disaster and propose recommendations to optimise systems performance [20]. These insights provide an evidence-base for the development of policies that support the improvement of future disaster response processes [21].

Stage 2 - Disseminate. Due to the infrequent nature of major natural disasters it is important that the lessons learned from one disaster response are disseminated effectively. However, the literature indicates that lessons are rarely communicated to their intended audience and that processes to promote the dissemination and uptake of lessons are weak [18]. Widespread dissemination of reports would ensure that the lessons learned during one disaster could be shared and used to improve disaster response practices for future events.

Stage 3 - Implement. The final stage of the M\&E cycle is to use the lessons identified in the report to design and implement changes to improve disaster response protocols. While there is much attention given to the 
need to continually improve response efforts, research indicates that minimal resources are invested into creating the capacity for improvement [22]. Consequently, it has been found that many health departments have failed to act on lessons identified during previous disasters [23].

\section{Search for primary studies}

The initial systematic search of the literature focussed on retrieving papers that investigated how health departments use M\&E to improve disaster response efforts. A search strategy was developed which focused on three main concepts; health departments; M\&E; and disaster response (Table 1 and Fig. 2). During the preliminary stages of developing the search strategy, we found very few papers examining how local health departments reflect on and analyse their response to a disaster. However, there appeared to be a significant degree of research on public health emergency preparedness. We postulated that many of the successes and challenges that local health departments experience when monitoring and evaluating the quality of emergency preparedness would be similar to the monitoring and evaluation of response efforts. Therefore, we included the term "preparedness" in our search strategy to enhance the body of literature to be analysed.

The same search strategy was used to explore electronic databases (i.e., PubMed, Embase, Scopus and Web of Science), in addition to Google and Google Scholar to retrieve any grey literature. We restricted our search from the year 2000 to the present as our preliminary scoping indicated that the majority of the literature describing the role of health departments in disasters emerged after the terrorist attacks on the United States in September 2001. There were no restrictions applied to the type of publication, however we only included papers written in English. The database searches concluded in May 2015 once a saturation point was reached (i.e., when the literature no longer provided new knowledge or added to our understanding) [19].

Consistent with realist evaluation our research involved collaboration with a local reference group who provided input throughout the review process. This local reference group included partners from the Philippines with expertise in disaster management. These partners facilitated the

Table 1 Search terms used

Disaster

AND quality OR monitoring OR evaluation OR "after action report" OR "after-action-report" OR "after action review" OR "after-action-review" OR

"after event report" OR "after-event-report" OR "after event review" OR

"after-event-review" OR drills

AND response OR recovery OR preparedness OR management

AND Health efficient identification of pertinent documents that would aid our research including disaster management policies and guidelines currently in use by health departments in the Philippines.

\section{Selection and quality appraisal of studies}

An inclusion criteria was established after discussions with the local reference group about what would be most useful in terms of M\&E for local health departments in the Philippines (Table 2). All titles and abstracts were independently assessed by two reviewers. Full text papers were retrieved and initially examined by one author to ensure that inclusion criteria were met. A second author then cross checked the selected papers before they were approved for review.

In realist evaluation, quality appraisal takes place during the synthesis stage and is guided by the concepts of relevance and rigour to determine whether potential information is fit for purpose $[18,19,24]$. When sifting through extracted information we first considered whether the information was relevant - i.e., does this information aid our understanding of potential mechanisms? Secondly we made judgments on whether the information provided sufficiently detailed descriptions of potential mechanisms - i.e., does this information contain rich detail that points to the mechanisms which link context and outcome?

The most relevant and detailed evidence featured more heavily in our analysis than papers containing weaker contributions. Realist evaluations do not typically apply a formal tool or checklist to assess potential biases. We did, however, consider elements of methodological soundness to aid in our quality appraisal (e.g. research question, study design, sample description, data collection procedure and data analytic technique). We included information contained within opinion pieces if such information was supported by or consistent with the findings of other empirical papers.

\section{Extraction, analysis and synthesis of relevant data}

One reviewer read each paper and recorded the following details in an extraction template: country setting; level of health system under investigation; type of disaster; stage of the theoretical framework being evaluated (document \& assess, disseminate and implement); and the barriers and facilitators of M\&E. During this stage, we identified recurring contextual themes across papers. To further investigate these themes, each paper was reread and important information regarding each contextual theme was transposed into another data extraction template. In this second iteration of data extraction we aimed to identify any prominent recurring patterns of contexts and outcomes, known as demi-regularities [18]. 


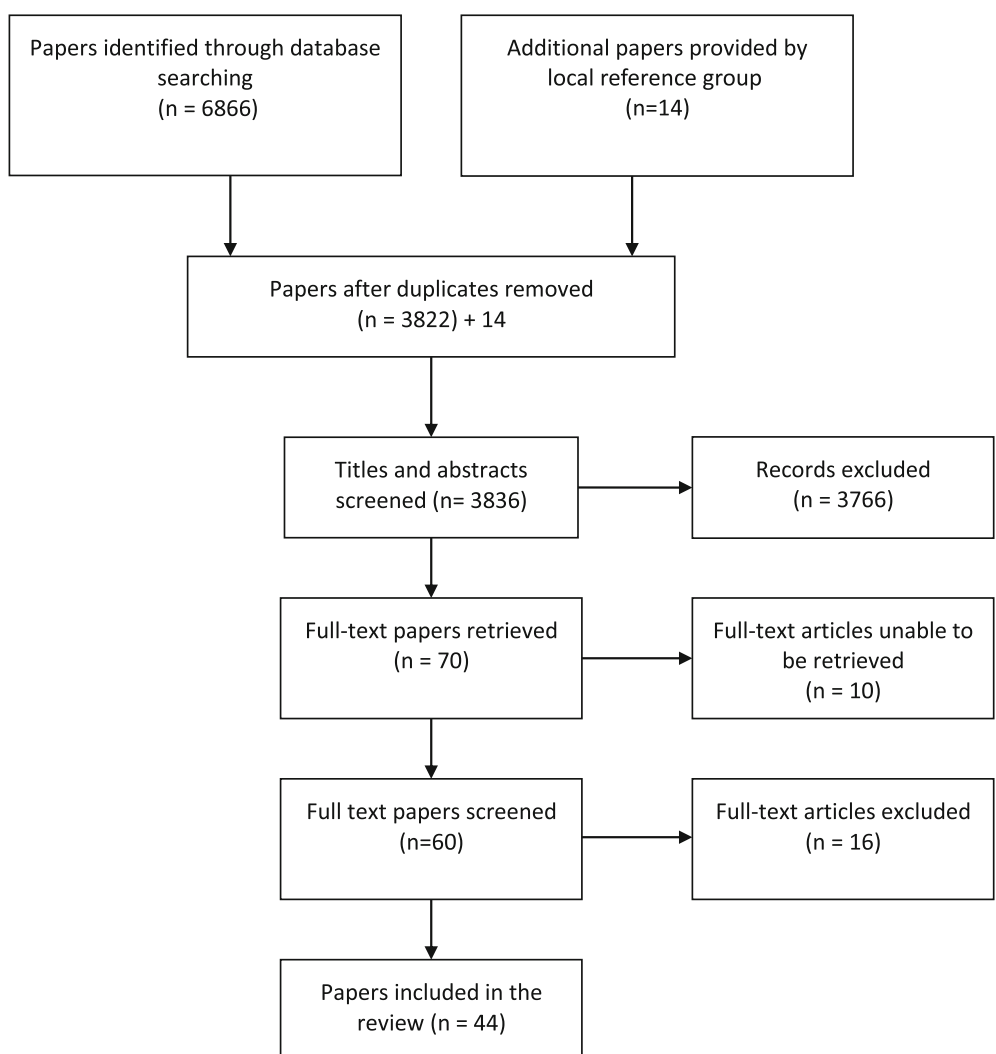

Fig. 2 Search Strategy

For example, we found that when health departments assure personnel that they will not be penalised for mistakes $(C)$ the documentation and assessment of a disaster response is likely to be more accurate and thorough $(\mathrm{O})$. We synthesised the extracted information to generate $\mathrm{CMO}$ configurations which explain causal pathways whereby certain contexts influence mechanisms that trigger positive or negative outcomes at each stage of M\&E. In this particular example, our synthesis revealed that when health departments assure personnel they will not be penalised for mistakes $(\mathrm{C})$ an organisational culture of learning is likely to be established which encourages staff motivation and willingness to engage with $M \& E(M)$. This would, in turn, result in more accurate and thorough documentation and assessment of the disaster response (O). We repeated this process of investigation and deduction for each of the demi-regularities found within the literature. Emerging findings were regularly shared and discussed with the review team to ensure the validity of inferences made.

\section{Refinement of the theoretical framework}

In realist reviews the synthesis of information is used to refine the theoretical framework to determine what

Table 2 Inclusion criteria

\begin{tabular}{l} 
Inclusion \\
\hline Papers which examined at least one stage of the theoretical framework \\
(document and assess; disseminate; or implement)
\end{tabular}

Papers which examined the evaluation of quality improvement efforts at the hospital level were included if their content was deemed to be applicable to health departments in general

\section{Exclusion}

Papers which focused on instruments used for the evaluation or adoption of performance standards, which are highly specific to the hospital setting

Papers which focused on evaluating elements of preparedness (i.e. facilities or training of health personnel) or were reporting the health outcomes after a disaster rather than the response itself

Papers which focused on research methods and metrics for the evaluation of disaster management and did not explore programme or policy aspects of the evaluation

Papers which reported the results of an evaluation but did not provide a description of how the evaluation was conducted, or how the lessons learned were disseminated or incorporated into future plans 
works for whom, how and under what circumstances [19]. We used the evidence synthesised in the review to fine tune our understanding of how M\&E can be successfully used to improve future disaster response efforts.

\section{Results \& findings}

Description of primary studies included in the analysis

Of the 44 papers included in this review, 23 made a significant contribution to our understanding of $\mathrm{CMO}$ configurations. Our quality assessment found that while some papers contained important contextual information regarding the current state of M\&E used in disasters, they did not include any relevant information that could be analysed against our theoretical framework. These papers were deemed to possess insufficient detail to be included in the evidence synthesis. However, these papers were not excluded from the full review as the underlying contextual information contained in these studies aided our understanding of the current context of M\&E approaches that have been used by health departments.

The following sections describe the $\mathrm{CMO}$ configurations identified within the demi-regularities in each stage of the M\&E cycle (a summary of the CMO configurations is presented in Table 3).

\section{Demi-regularities identified in the 'document and assess' stage of the monitoring and evaluation cycle}

There were three demi-regularities identified within the document and assess stage of the M\&E cycle.

The first demi-regularity in the document and assess stage was, "When health personnel are assured that there will be no punitive action for reporting mistakes they are more likely to accurately document the disaster response'. A number of seminal papers identified through this review highlighted that health personnel are reluctant to formally acknowledge and document errors made during a disaster response due to potential ramifications (e.g., liability, concerns about future funding and career advancement) $[13,25,26]$. However, concealing such errors leads to an inaccurate representation of the response and hinders improvement efforts. Seid and colleagues [22] found that an organisational culture which fosters openness, collaboration, teamwork, and learning from mistakes to be optimal for quality improvement.

When evaluation reports are driven by quality improvement purposes, health personnel are more likely to identify the potential pitfalls which may adversely affect a response to a future event [14]. We infer that within the context of a supportive and open workplace a culture of learning is instilled within the health department. This mechanism is likely to result in more accurate documentation and assessment of the disaster response as health personnel feel comfortable to report errors.

The second demi-regularity in the document and assess stage was, 'Embedded training leads to more accurate documentation and assessment of disaster responses'. Accurately documenting and assessing a response to a disaster involves identifying strengths and weaknesses in the response and devising corrective actions which may improve future performance [13]. Due to the infrequency of large-scale disasters, health personnel have limited opportunities to learn and practice the analytical skills required to accurately document a response. This limitation may explain the lack of in-depth, quality insights contained in evaluation reports that have been acknowledged in the literature $[13,27]$. Several of the key papers identified in this review noted that embedded training has proven to be a useful method for familiarising staff with

Table 3 Context-Mechanism-Outcome configurations

\begin{tabular}{|c|c|c|c|}
\hline Stage of M\&E & Context & Mechanism & Outcome \\
\hline \multirow[t]{3}{*}{ Document and assess } & $\begin{array}{l}\text { Assurance that staff will not be } \\
\text { penalised for mistakes. }\end{array}$ & $\begin{array}{l}\text { A culture of learning is instilled in the } \\
\text { health department }\end{array}$ & $\begin{array}{l}\text { More accurate and high quality } \\
\text { documentation and assessment } \\
\text { of disaster response }\end{array}$ \\
\hline & $\begin{array}{l}\text { Training in the procedures and skills for } \\
\text { documenting and assessing a disaster } \\
\text { response are embedded into regular work }\end{array}$ & $\begin{array}{l}\text { Provides staff with an opportunity to } \\
\text { practice and become proficient in the } \\
\text { analytical skills required to successfully } \\
\text { document and assess their response. }\end{array}$ & Better prepared staff \\
\hline & $\begin{array}{l}\text { Using external partners to document and } \\
\text { assess the disaster response }\end{array}$ & $\begin{array}{l}\text { External partners have the time, knowledge } \\
\text { and skills to thoroughly document and } \\
\text { assess a response }\end{array}$ & $\begin{array}{l}\text { Improved depth and quality of } \\
\text { documentation and assessment }\end{array}$ \\
\hline Disseminate & Disseminating standardised reports & $\begin{array}{l}\text { Allows all health departments to perceive } \\
\text { the experiences of others as relevant to } \\
\text { their own work }\end{array}$ & Promotes inter-agency learning \\
\hline \multirow[t]{2}{*}{ Implement } & Positive leadership attributes & $\begin{array}{l}\text { Establishes a culture of quality improvement } \\
\text { within the health department which increases } \\
\text { commitment }\end{array}$ & $\begin{array}{l}\text { Promotes successful } \\
\text { implementation of changes } \\
\text { to improve performance }\end{array}$ \\
\hline & Shared decision making structures & $\begin{array}{l}\text { Fosters a sense of ownership and responsibility } \\
\text { amongst personnel which increases motivation } \\
\text { and commitment }\end{array}$ & $\begin{array}{l}\text { Promotes successful } \\
\text { implementation of changes } \\
\text { to improve performance }\end{array}$ \\
\hline
\end{tabular}


the procedures that should be enacted when responding to a disaster situation $[3,22,23,28]$.

For example, in Nelson et al. [23], one of the most informative papers of this review, it was recounted that some public health departments across the United States have used annual influenza clinics to practice mass dispensing procedures, which may be required in a disaster situation [23]. Similarly, local health departments could use embedded training to provide personnel with opportunities to practice documenting the delivery of routine health services and considering how procedures may be improved. This process would train personnel to be proficient in the analytical skills required to successfully document and assess a disaster response. This mechanism is likely to result in more accurate and thorough documentation and assessment of future disaster response efforts.

The third demi-regularity in the document and assess stage was, 'Partnering with external organisations improves the depth and quality of documentation and assessments'. In the aftermath of a disaster, health personnel are under extreme pressure to deliver emergency health services, minimise disruptions to regular health services and generate evaluation reports $[29,30]$. The literature indicates that assessments conducted immediately after a disaster or after the termination of an emergency drill, are more likely to capture accurate narrative information that may otherwise be lost over time $[9,20,31]$. Some health departments have begun to partner with external organisations to alleviate the administrative burden on health personnel and to ensure that information is collected and analysed efficiently [3, 29, 32-34]. External organisations may include local academic institutions or neighbouring health departments who have the time, skills and necessary knowledge to accurately document and assess a disaster response. The analytical skills of the external partners acts as the mechanism by which to produce thorough documentation and objective assessments of a health department's response to a disaster [26, 33].

\section{Demi-regularities identified in the 'disseminate' stage of the monitoring and evaluation cycle}

There was one demi-regularity identified within the disseminate stage of the M\&E cycle. This demi-regularity can be summarised as follows, 'The standardisation of M\&E reports is likely to promote inter-agency learning'. The broad dissemination of evaluation reports is critical to building a body of evidence from which all health departments can learn. However, there is currently a high degree of variability in the structure and terminology used in evaluation reports, alongside gaps in the quality and completeness of data [13, 35, 36]. Such inconsistencies make it difficult for one health department to perceive the experiences of another as relevant to their own work $[4,13]$. The dissemination of standardised reports would allow all health departments to acknowledge how the lessons learned by another department may be applicable to their own. This mechanism would: support comparisons across settings and over time; promote inter-agency learning; and help health departments to continually refine their response procedures $[23,26]$.

\section{Demi-regularities identified in the 'implement' stage of the monitoring and evaluation cycle}

There were two demi-regularities identified within the implement stage of the M\&E cycle.

The first demi-regularity in the implement stage was, 'Positive leadership attributes at the management level increases the likelihood that changes to improve response procedures will be successfully implemented'. The evidence synthesised in this RRR clearly demonstrates that well-defined leadership is critical to the success of implementation activities. We found substantial evidence from the literature which supported the notion that leaders who foster cooperation, value input from staff, empower employees, are enthusiastic and provide constructive feedback are crucial to facilitating an organisational culture of quality improvement $[22,37,38]$. We postulate that the establishment of a culture of quality improvement is a mechanism that can increase motivation to refine disaster response processes.

The second demi-regularity in the implement stage was, 'Shared decision making structures are likely to promote successful implementation processes'. Creating sustainable change in an emergency response organisation can be a highly complex and difficult task [13, 22]. We found that decision making structures play a significant role in influencing how health personnel respond to activities and also to organisational changes. Involving health personnel when allocating roles and responsibilities in disaster scenarios facilitates a sense of equity, ensures buy-in and also encourages a sense of responsibility [28]. Furthermore, involving health personnel in planning implementation activities ensures that specific needs and resources are considered and that the proposed changes are achievable $[3,13,34]$. This context of shared decision making triggers a mechanism that fosters a sense of ownership amongst health personnel. This, in turn, facilitates the successful adoption and execution of implementation activities by health personnel.

\section{Discussion}

\section{Summary of findings}

Local health departments are often at the forefront of a disaster response delivering lifesaving health services. M\&E can be used to help health departments reflect on their response efforts to learn from what worked and what did not and to identify ways to improve their 
response to future events. However, the literature indicates that many health departments struggle to effectively learn from past mistakes, thus the same errors are often repeated time and again $[4,12-15]$. We conducted this RRR to understand how health departments can better use M\&E to successfully learn from past experiences and improve disaster response procedures.

In the first stage of our M\&E cycle, document and assess, we uncovered several CMO configurations which were likely to encourage thorough documentation and assessment of the disaster response. Firstly, there was strong support in the literature for health departments to assure staff that they would not be penalised for mistakes. This was found to create a culture of learning whereby staff felt comfortable to admit to mistakes and report response errors. This mechanism would lead to more accurate documentation of the response. Secondly, embedded training methods provide health personnel with regular opportunities to practice documenting and assessing their work. This leads to staff being more familiar with documentation procedures and is likely to translate into higher quality documentation and assessment of a disaster response. Alternatively, using external partnerships is likely to improve the depth and quality of reports as such partners have the necessary skills to document and assess a response.

Dissemination is a crucial component of M\&E which promotes the sharing of lessons to a broad range of stakeholders. However, the literature indicated that dissemination was one of the weakest points in the application of M\&E in the disaster context. Despite this recurrent finding, there appeared to be very limited research into why this was the case and how dissemination could be improved. Due to this gap in the literature, we were only able to analyse one demi-regularity. This mechanism works by allowing personnel from one department to understand and perceive the experiences of others as relevant to their own. Such a mechanism would be triggered when the disseminated reports are standardised in both format and terminology, thereby promoting inter-agency learning. It is acknowledged that this mechanism is heavily influenced by factors external to a health department. For example, there would need to be a governing body who oversees the dissemination of all reports to ensure they are written to standard. This would require a sustained commitment and administrative effort which not all governments will have the capacity for. Further research into how health departments in LMICs can broadly disseminate their lessons is needed. At the implementation stage of the M\&E cycle, positive leadership attributes and shared decision making structures were both found to promote successful outcomes by motivating staff and also fostering a sense of responsibility and ownership among health personnel.
This RRR revealed that there are very few studies which have investigated experiences with $M \& E$ in LMICs, despite these countries being disproportionately affected by disasters. This is consistent with the more general findings of Gocotano and colleagues (2015) who found that there are fewer papers documenting disaster or emergency events in low-income countries compared to high-income countries [39]. Only 2 of the 30 papers included in this RRR analysed the experiences of health departments in LMICs [20,38], while the remaining literature focused on high-income countries (the United States in particular). It has recently been noted that even when papers are published on events in low-income countries, they are often written by authors with institutional affiliations external to the country of the event [39]. These factors may mean that there are CMO configurations unique to the experience of local personnel working in health departments in low resource countries which are not adequately reflected in the literature we have analysed. Although many of the CMO configurations synthesised in this RRR have been drawn from literature on disaster and emergency health events in high-income countries, the findings are applicable and transferrable to other setting due to the 'all-hazard approach' in disaster management, which emphasises that important systems issues will be common across disasters [21].

\section{Strengths and limitations}

A strength of this RRR lies in its utilisation of realist methodology, in particular the use of the CMO configurations, to understanding not only whether an intervention works or not but also how and why an intervention (i.e., M\&E) may work. Other evidence synthesis approaches used for informing policy decisions, such as meta-analyses or narrative studies, have key limitations which prevent an explanation of how an intervention works and how it may operate in a different setting [16]. This RRR used a heuristic approach to examine the existing literature describing health departments' experiences with M\&E. A synthesis of this evidence identified a number of mechanisms that may facilitate or hinder the M\&E process. The identification of these mechanisms is in itself of great value to health departments implementing M\&E. However further value is gained from this RRR through the theory driven and explanatory nature of realist evaluation. This approach enabled the construction of CMO configurations that explain exactly how and why M\&E can be successfully used to learn lessons and improve future disaster response efforts. Although available evidence lacked sufficient detail for us to conduct a more comprehensive examination of what works and does not work at each stage of the disaster recovery cycle, we were able to draw lessons that can be applied across the board. 
A possible limitation of this review is that some of the $\mathrm{CMO}$ configurations identified are, at this stage, based on the recommendations or ideas of the original authors' of the reviewed papers, rather than tested experiences of M\&E. This may reflect either a shortage of health departments engaging with M\&E to learn lessons from disaster response efforts or a gap in research investigating health departments' experiences with M\&E. Nevertheless, the inferences drawn in this RRR are clearly acknowledged and based on a rigorous analysis of the literature currently available. We have aimed for transparency by documenting which papers contributed to our synthesis of $\mathrm{CMO}$ configurations (Table 4) and advise readers to carefully consider how these $\mathrm{CMO}$ configurations relate to their situation.

Table 4 Papers which contributed to final analysis

\begin{tabular}{|c|c|c|c|c|}
\hline $\begin{array}{l}\text { Stage of theoretical } \\
\text { framework }\end{array}$ & Document reference & Country setting & Type of disaster & Level of health system \\
\hline \multicolumn{5}{|c|}{ Document and Assess } \\
\hline & Green et al. 2003 [20] & Guatemala & $\begin{array}{l}\text { Disaster scenario - propane tank } \\
\text { explosion at an open-air food vendor }\end{array}$ & Hospital \\
\hline & Klein et al. 2005 [34] & USA & Bioterrorism & Hospital \\
\hline & Macrae 2014 & UK & Healthcare disasters & Hospital \\
\hline & Seale 2010 [28] & USA & Hurricanes (Rita 2005, Ike 2008) & $\begin{array}{l}\text { Post-acute rehabilitation } \\
\text { facility }\end{array}$ \\
\hline & Biddinger et al. 2008 [32] & USA & All public health emergencies & $\begin{array}{l}\text { State and local public health } \\
\text { departments }\end{array}$ \\
\hline & Nelson, Lurie \& Wasserman 2007 [23] & USA & All disasters & Public health system \\
\hline & Piltch-Loeb 2014a [26] & USA & All disasters & Public health system \\
\hline & Piltch-Loeb 2014b [33] & USA & All disasters & Public health system \\
\hline & Savoia, Agboola \& Biddinger 2012 [14] & USA & $\begin{array}{l}\text { H1N1 pandemic and hurricanes } \\
\text { (Ike 2008, Gustav 2008, Katrina 2005) }\end{array}$ & Public health system \\
\hline & Seid et al. 2007 [22] & USA & All disasters & Public health system \\
\hline & Stebbins \& Vukotich 2010 [29] & USA & Hepatitis A outbreak & Public health department \\
\hline & Adini et al. 2013 [3] & Israel & Mass casualty incidents & National health system \\
\hline & ảBirkland 2009 [30] & USA & All disasters & Not health specific \\
\hline & ãDonohue \& Tuohy 2006 [13] & USA & All disasters & Not health specific \\
\hline & aDufty 2013 [31] & Australia & All emergencies & Not health specific \\
\hline & aSpillsbury et al. 2007 [27] & Not country specific & Not disaster specific & Not health specific \\
\hline \multicolumn{5}{|l|}{ Disseminate } \\
\hline & Mears et al. 2010 [35] & USA & All public health emergencies & State public health system \\
\hline & Nelson, Lurie \& Wasserman 2007 [22] & USA & All disasters & Public health system \\
\hline & Piltch-Loeb 2014a [26] & USA & All disasters & Public health system \\
\hline & Savoia, Preston \& Biddinger 2013 [36] & USA & All public health emergencies & Public health system \\
\hline & ªnohue \& Tuohy 2006 [13] & USA & All disasters & Not health specific \\
\hline \multicolumn{5}{|l|}{ Implement } \\
\hline & Adini et al. 2012 [21] & Israel & Mass casualty events & Hospital \\
\hline & Klein et al. 2005 [34] & USA & Bioterrorism & Hospital \\
\hline & Bevc et al. 2012 [37] & USA & All disasters & Local health department \\
\hline & Chan et al. 2010 [38] & Indonesia & Tsunami & Local health clinics \\
\hline & Seale 2010 & USA & Hurricanes (Rita 2005, Ike 2008) & $\begin{array}{l}\text { Post-acute rehabilitation } \\
\text { facility }\end{array}$ \\
\hline & Seid et al. 2007 [22] & USA & All disasters & Public health system \\
\hline & Adini et al. 2013 [3] & Israel & Mass casualty incidents & National health system \\
\hline & aatalden \& Davidoff 2007 & Not country specific & Not disaster specific & Healthcare systems \\
\hline & ãDonohue \& Tuohy 2006 [13] & USA & All disasters & Not health specific \\
\hline
\end{tabular}


During the search process, we excluded non-health research to ensure that we would only evaluate issues relevant to health departments when conducting M\&E. However, several papers provided by the local reference group focussed on other sectors, particularly high-risk industries such as aviation, which have made significant progress with quality improvement approaches such as M\&E $[13,22,30,33]$. We suggest that future research consider how other sectors have been able to refine their M\&E practices. Investigation outside of the health sector could reveal transferable theories that may assist local health departments to improve M\&E practices.

\section{Conclusion}

Despite the sparse literature, it was evident that local health departments who engage with $M \& E$ processes to evaluate their responses to major disasters often fail to improve future response efforts. This paper summarises the CMO configurations that were found to have an effect on outcomes at each stage of the M\&E cycle (document and assess, disseminate and implement). We have considered how these may act to constrain or enable the ability of people using M\&E approaches to effectively learn lessons from previous experiences. Firstly, to support thorough and accurate documentation and assessment of a disaster response, local health departments should consider how they can: create a culture of learning within health departments; use embedded training methods; or facilitate external partnerships. Secondly, to enhance the widespread dissemination of lessons learned from a disaster response effort, evaluation reports should be standardised in format and terminology. Lastly, to increase commitment to the implementation of improvement processes, local health department leaders should display positive leadership attributes and encourage shared decision making.

Further research relevant to disaster prone regions is critical to enabling additional $\mathrm{CMO}$ configurations to be uncovered and to contribute to the understanding of how local health departments can use M\&E to effectively learn from the past. For example, there was some emerging evidence [13, 26, 29] which suggested that digital platforms could be used to assist in the broad dissemination of lessons learned from disaster response efforts. Further exploration of this idea was hindered by the paucity of evidence in the literature. Future research should analyse how new technologies may be utilised in M\&E to improve disaster response efforts.

This study is among the first to conduct a synthesis of the CMO configurations which facilitate or hinder M\&E efforts aimed at improving future disaster responses. It makes a significant contribution to the disaster literature and provides an evidence base to help health departments to understand how they may successfully use M\&E to improve disaster response efforts. Our findings and recommendations will be particularly useful for health departments in LMICs of East Asia and the Pacific who are frequently affected by disasters and stand to gain the most from rigorous and purposeful disaster M\&E.

\begin{abstract}
Abbreviations
CMO: Context-mechanism-outcome; LMIC: Low and middle income country; M\&E: Monitoring and evaluation; RRR: Rapid realist review

\section{Acknowledgments}

None.

Funding

This research was supported by UNICEF, grant number SM149910.

\section{Availability of data and materials}

The qualitative data set collated during this rapid realist review is available upon request.

\section{Authors' contributions}

The research was conceived and designed by KG and E. Searches were conducted by KG and E. Data was extracted by KG in consultation with EJ and SF. Data was analysed by KG in consultation with E. The manuscript was prepared by KG with input from all authors. All authors provided critical commentary on earlier versions of the manuscript, and read and approved the final manuscript.
\end{abstract}

Ethics approval and consent to participate Not applicable.

\section{Consent for publication}

Not applicable.

\section{Competing interests}

The authors declare that they have no competing interests.

\section{Publisher's Note}

Springer Nature remains neutral with regard to jurisdictional claims in published maps and institutional affiliations.

\section{Author details}

${ }^{1}$ School of Public Health, The University of Queensland, Level 2, Public Health Building (887) Corner of Herston Road and Wyndham Street, Herston QLD 4006, Australia. ${ }^{2}$ Policy and Epidemiology Group, Queensland Centre for Mental Health Research, The Park Centre for Mental Health, Level 3, Dawson House, Wacol QLD 4076, Australia. ${ }^{3}$ Global Burden of Disease Group Melbourne School of Population and Global Health, The University of Melbourne, Level 5, Building 379, 207 Bouverie Street, Carlton, VIC 3010, Australia. ${ }^{4}$ UNICEF Philippines Country Office, 31st Floor, Yuchengco Tower Rizal Commercial Banking Corporation (RCBC) Plaza 6819 Ayala Avenue corner Gil Puyat Avenue Makati City, 1200 Makati, Philippines. ${ }^{5}$ Institute of Tropical Medicine Antwerp, Nationalestraat 155, 2000 Antwerp, Belgium. ${ }^{6}$ Department of Obstetrics and Gynecology, Medical University of Graz, Auenbruggerplatz 14, A-8036 Graz, Austria. ${ }^{7}$ Abt Associates Australia, 5 Gardner Cl, Milton QLD, Brisbane 4064, Australia.

Received: 18 December 2016 Accepted: 19 June 2017

Published online: 29 June 2017

\section{References}

1. United Nations. Sendai framework for disaster risk reduction 2015-2030. 2015

2. Jha AK, Stanton-Geddes Z. Strong, safe, and resilient: a strategic policy guide for disaster management in East Asia and the Pacific. 2013.

3. Adini B, Peleg K. On constant alert: lessons to be learned from Israel's emergency response to mass-casulty terrorism incidents. Health Aff. 2013; 32(12):2178-85.

4. Birnbaum ML, Daily EK, O'Rourke AP, Kushner J. Research and evaluations of the health aspects of disasters, Part VI: interventional research and the disaster logic model. Prehosp Disaster Med. 2016;31(2):181-94. 
5. Birnbaum ML, Daily EK, O'Rourke AP. Research and evaluations of the health aspects of disasters, Part IV: framework for societal structures: the societal systems. Prehosp Disaster Med. 2015;30(6):633-47.

6. Birnbaum ML, Daily EK, O'Rourke AP. Research and evaluations of the health aspects of disasters, Part III: framework for the temporal phases of disasters. Prehosp Disaster Med. 2015;30(6):628-32.

7. Birnbaum ML, Daily EK, O'Rourke AP. Research and evaluations of the health aspects of disasters, Part VII: the relief/recovery framework. Prehosp Disaster Med. 2016;31(2):195-210.

8. Birnbaum ML, Daily EK, O'Rourke AP, Loretti A. Research and evaluations of the health aspects of disasters, Part II: the disaster health conceptual framework revisited. Prehosp Disaster Med. 2015;30(5):523-38.

9. Miller KA. Emergency management in health care: an all-hazards approach 2nd ed. The Joint Commission: USA; 2012

10. Young LL. Evaluation methods for assessing public health medical response to disasters Younf Landesman L, editor. Washington D.C.: American Public Health Association; 2012

11. ProVention Consortium. Evaluating disaster risk reduction initiatives. 2007.

12. Auf der Heide $E$. The importance of evidence-based disaster planning. Ann Emerg Med. 2006;47(1):34-49.

13. Donohue AK, Tuohy RV. Lessons we don't learn: a study of the lessons of disasters, why we repeat them, and how we can learn them. 2006.

14. Savoia E, Agboola F, Biddinger PD. Use of after action reports (AARs) to promote organizational and systems learning in emergency preparedness. Int J Environ Res Public Health. 2012;9(8):2949-63.

15. World Bank. Building resilient communities: risk management and response to natural disasters through social funds and community-driven development operations. 2009.

16. Saul JE, Willis CD, Bitz J, Best A. A time-responsive tool for informing policy making: rapid realist review. Implement Sci. 2012;8(103):15.

17. Pawson R. Evidence-based policy: a realist perspective London: sage publications 2006.

18. Wong G, Greenhalgh T, Westhorp G, Buckingham J, Pawson R. RAMESES publication standards: realist syntheses. BMC Med. 2013;11:21.

19. Pawson R, Greenhalgh T, Harvey G, Walshe K. Realist review - a new method of systematic review designed for complex policy interventions. J Health Serv Res Policy. 2005;10(1):13.

20. Green GB, Modi S, Lunney K, Thomas TL. Generic evaluation methods for disaster drills in developing countries. Ann Emerg Med. 2003;41:689-99.

21. Adini B, Laor D, Hornik-Lurie T, Schwartz D, Aharonson-Daniel L. Improving hospital mass casualty preparedness through ongoing readiness evaluation. Am J Med Qual. 2012;27(5):426-33.

22. Seid M, Lotstein D, Williams VL, Nelson CD, Leuschner KJ, Diamant A, et al. Quality improvement in public health emergency preparedness. Annu Rev Public Health. 2007;28:19-31.

23. Nelson C, Lurie N, Wasserman J. Assessing public health emergency preparedness: concepts, tools and challenges. Annu Rev Public Health. 2007; 28:1-18.

24. Windle G, Gregory S, Newman A, Goulding A, O'Brien D, Parkinson C. Understanding the impact of visual arts interventions for people living with dementia: a realist review protocol. Syst Rev. 2014;3:91.

25. Macrae C. Early warnings, weak signals and learning from healthcare disasters. BMJ Qual Saf. 2014;23:440-5.

26. Piltch-Loeb RN, Kraemer JD, Nelson CD, Stoto MA. A public health emergency prepardeness critical incident registry. Biosecur Bioterror. 2014a; 12(3):132-43.

27. Spillsbury MJ, Perch C, Norgbey S, Rauniyar G, Battaglino C. Lessons learned from evaluation: a platform for sharing knowledge. 2007.

28. Seale GS. Emergency preparedness as a continuous improvement cycle: perspectives from a post-acute rehabilitation facility. Rehabil Psychol. 2010; 55(3):247-54.

29. Stebbins S, Vukotich CJ. Preserving lessons learned in disease outbreaks and other emergency responses. Aust J Public Health. 2009;32(4):467-71.

30. Birkland TA. Disasters, lessons learned and fantasy documents. J Conting Crisis Manag. 2009;17(2):146-56

31. Dufty N. Evaluating emergency management after an event: gaps and suggestions. Aust J Emerg Manage. 2013;28(4):15-9.

32. Biddinger PD, Cadigan RO, Auerbach BS, Burstein JL, Savoia E, Stoto MA, et al. Using exercises to identify systems level preparedness challenges. Public Health Rep. 2008;123(1):96-101.
33. Piltch-Loeb RN, Nelson CD, Kraemer JD, Savoia E, Stoto MA. A peer assessment approach for learning from public health emergencies. Public Health Rep. 2014b;129(4):28-34.

34. Klein KR, Bradenburg DC, Atlas JG, Maher A. The use of trained observers as an evaluation tool for a multi-hospital bioterrorism exercise. Prehosp Disaster Med. 2005;20(3):159-63.

35. Mears GD, Drexdal P, Glickman SW, Brice JH, Glickman LT, Cabanas JG. The North Carolina EMS data system: a comprehensive intergrated emergency medical services quality improvement program. Prehosp Emerg Care. 2010; 14:85-94.

36. Savoia E, Preston J, Biddinger PD. A consensus process on the use of exercises and after action reports to asses and improve public health emergency preparedness and response. Prehosp Disaster Med. 2013;28(3): 305-8.

37. Bevc CA, Simon MC, Montoya TA, Horney JA. Institutional facilitators and barriers to local public health preparedness planning for vulnerable and atrisk populations. Public Health Rep. 2014;129:35-41.

38. Chan GJ, Parco KB, Sihombing ME, Tredwell SP, O'Rourkea EJ. Improving health services to displaced persons in Aceh, Indonesia: a balanced scorecard. Bull World Health Organ. 2010;88:709-12.

39. Goctano A, Counahan M, Belizario V, Hartigan-Go K, Balboa G, Go M, et al. Can you help me write my story? The institutional affiliations of authors of international journal articles on post-disaster health response. Western Pac Surveill Response J. 2015;6:10-4.

\section{Submit your next manuscript to BioMed Central and we will help you at every step:}

- We accept pre-submission inquiries

- Our selector tool helps you to find the most relevant journal

- We provide round the clock customer support

- Convenient online submission

- Thorough peer review

- Inclusion in PubMed and all major indexing services

- Maximum visibility for your research

Submit your manuscript at www.biomedcentral.com/submit
Biomed Central 